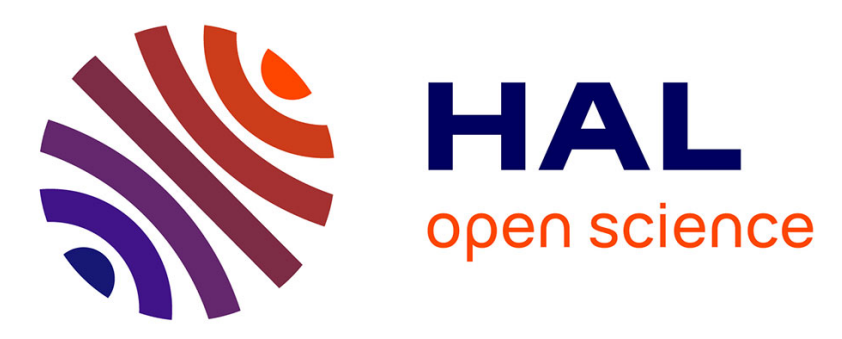

\title{
Interfacial waves between piezoelectric and piezomagnetic half-spaces with magneto-electro-mechanical imperfect interface
}

J.A. Otero, Reinaldo Rodriguez-Ramos, G. Monvisais, C. Stern, Frédéric Lebon

\section{To cite this version:}

J.A. Otero, Reinaldo Rodriguez-Ramos, G. Monvisais, C. Stern, Frédéric Lebon. Interfacial waves between piezoelectric and piezomagnetic half-spaces with magneto-electro-mechanical imperfect interface. Philosophical Magazine Letters, 2013, 93 (7), pp.413-421. 10.1080/09500839.2013.793850 . hal-00861443

\section{HAL Id: hal-00861443 https://hal.science/hal-00861443}

Submitted on 19 Jun 2018

HAL is a multi-disciplinary open access archive for the deposit and dissemination of scientific research documents, whether they are published or not. The documents may come from teaching and research institutions in France or abroad, or from public or private research centers.
L'archive ouverte pluridisciplinaire HAL, est destinée au dépôt et à la diffusion de documents scientifiques de niveau recherche, publiés ou non, émanant des établissements d'enseignement et de recherche français ou étrangers, des laboratoires publics ou privés. 


\title{
Interfacial waves between piezoelectric and piezomagnetic half-spaces with magneto-electro-mechanical imperfect interface
}

\author{
J.A. Otero ${ }^{\mathrm{a} *}$, R. Rodríguez-Ramos ${ }^{\mathrm{b}}$, G. Monsivais ${ }^{\mathrm{c}}$, C. Stern ${ }^{\mathrm{d}}$ and F. Lebon ${ }^{\mathrm{e}}$ \\ ${ }^{a}$ Instituto de Cibernética, Matemática y Física (ICIMAF), 15 No. 551, Vedado, C.P. 10-400, La \\ Habana, Cuba, ${ }^{b}$ Facultad de Matemática y Computación, Universidad de La Habana, San Lazaro \\ y L, Vedado, C.P. 10-400, La Habana, Cuba; ${ }^{c}$ Instituto de Física, Universidad Nacional Autónoma \\ de México, A.P. 20-364, 01000 México, D.F, México: ${ }^{d}$ Facultad de Ciencias, Universidad Nacional \\ Autónoma de México, D.F., 04510, México; ${ }^{e}$ Laboratoire de Mécanique et d'Acoustique, Université \\ Aix-Marseille 1, 31 Chemin Joseph-Aiguier, 13402 Marseille Cedex 20, France
}

\begin{abstract}
We study the propagation of shear horizontal waves between the interface of piezoelectric and piezomagnetic half-spaces with a magneto-electro-mechanical imperfect contact. Mechanical, electrical and magnetical imperfections are modelled by means of a spring, a capacitor and a inductor, respectively. A general expression for the dispersion relation not reported previously in literature is given in an explicit form, with the diverse limit cases analysed in detail. In some of these limit cases, new expressions are also obtained which predict the existence of interfacial waves. In the other cases, when already reported results exist, a comparison with them is done. Some physical interpretations are derived from the limit cases. The influence of mechanical, electrical and magnetical imperfect contacts are shown in some numerical examples.
\end{abstract}

Keywords: interfacial wave; dispersion relation; imperfect bonding; piezoelectric; piezomagnetic

Recently, Yang Z. and Yang J. [1] and Nan et al. [2] studied the shear horizontal piezoelectric waves propagating along the interface between two semi-infinite half-spaces. The propagation of an interfacial shear wave in two bonded semi-infinite piezoelectric and piezomagnetic materials with a perfect interface has been studied by Soh and Liu [3]. The existence of certain waves propagating near an imperfectly bonded interface between two half-spaces of different piezoelectric ceramics has been studied by Fan, Yang and Xu [4]. In Ref. [4], imperfection is considered by means of a discontinuity of the displacement (spring model). The influence of elastic imperfect bonding on interface waves guided by piezoelectric/piezomagnetic composites has been presented by Melkumyan and Mai [5] and Huang et al. [6]. Besides, some other works related to interface waves in composite materials with elastic imperfect contact at the interface have been published by Darinskii and Weihnacht [7], Bai et al. [8]. The imperfect bonding sometimes exists in devices, but little is known about its influence on the wave propagations in it. Otero et al. [9] studied dispersion relations of surface $\mathrm{SH}$-waves in heterostructures with elastic imperfect bonding at the

*Corresponding author. Email: jaotero@icimaf.cu 
interface. The mechanical imperfection of the interface should also induce electric and/or magnetic defects in the interface piezoelectric/piezomagnetic due to their magneto-electromechanical coupling. More recently, the propagation of shear horizontal waves between the interface of two piezoelectric material with an electro-mechanical imperfect contact has been studied by Otero et al. [10]. Here, the mechanical and electrical imperfections have been modelled by means of a spring and capacitor ([11]), respectively, where the imperfect interface affects the interfacial wave.

In the present work, we have considered the existence of magnetic, electric and mechanic imperfections at the interface between piezoelectric and piezomagnetic half-spaces. The presence of magnetical [12], electrical [11] and mechanical imperfections are modelled by means of an inductor, a capacitor and a spring, respectively. The capacitance (inductance) of the capacitor (inductor) is a measure of the electrical (magnetical) imperfection.

Let us consider piezoelectric and piezomagnetic half-spaces with an interface. The $x z$ plane is the interface at $y=0$. The space $y>0$ is occupied by piezoelectric and the space $y<0$ by piezomagnetic. Both materials have $6 \mathrm{~mm}$ hexagonal symmetry and polarization in the $z$-axis direction. For the case of shear horizontal $(\mathrm{SH})$ waves, the displacement vector $\mathbf{u}=\left(u_{x}, u_{y}, u_{z}\right)$, the electric potential $\varphi$ and the magnetic potential $\psi$ are given by $u_{x}=u_{y}=0, u_{z}=u(x, y, t), \varphi=\varphi(x, y, t)$ and $\psi=\psi(x, y, t)$. Auxiliary functions $\bar{\varphi}$ and $\bar{\psi}$ can be introduced by $\varphi=\bar{\varphi}+e u / \varepsilon$ and $\psi=\bar{\psi}+f u / \mu$, where $e=e_{15}, f=f_{15}$, $\varepsilon=\varepsilon_{11}$ and $\mu=\mu_{11}$ are the piezoelectric, piezomagnetic, dielectric permittivity, magnetic permeability, respectively. The governing equations for $\mathrm{SH}$ waves in the half-spaces are [4-6]

$$
\begin{aligned}
& \bar{c}_{A} \nabla^{2} u_{A}=\rho_{A} \ddot{u}_{A}, \quad \nabla^{2} \bar{\varphi}_{A}=0, \quad \nabla^{2} \psi_{A}=0, \quad y>0, \\
& \bar{c}_{B} \nabla^{2} u_{B}=\rho_{B} \ddot{u}_{B}, \quad \nabla^{2} \varphi_{B}=0, \quad \nabla^{2} \bar{\psi}_{B}=0, \quad y<0,
\end{aligned}
$$

where $\bar{c}_{A}=c_{A}+e_{A}^{2} / \varepsilon_{A}, \bar{c}_{B}=c_{B}+f_{B}^{2} / \mu_{B}$ and $c=c_{44}$ is the elastic constant. $\nabla^{2} \equiv$ $\partial^{2} / \partial x^{2}+\partial^{2} / \partial y^{2}$ is the two-dimensional Laplacian. The subscript $A(B)$ indicate quantities in piezoelectric (piezomagnetic) half-space. For interfacial wave, it is required that $u, \phi$ and $\psi$ vanish at $y= \pm \infty$; which in turn implies that there are solutions of Equation (1) of the form

$$
\begin{array}{cc}
u_{A}=U_{A} \exp \left(-\eta_{A} y\right) \cos (\xi x-\omega t), & y>0, \\
\bar{\varphi}_{A}=\Phi_{A} \exp (-\xi y) \cos (\xi x-\omega t), & y>0, \\
\psi_{A}=\Psi_{A} \exp (-\xi y) \cos (\xi x-\omega t), & y>0, \\
u_{B}=U_{B} \exp \left(\eta_{B} y\right) \cos (\xi x-\omega t), & y<0, \\
\phi_{B}=\Phi_{B} \exp (\xi y) \cos (\xi x-\omega t), & y<0, \\
\bar{\psi}_{B}=\Psi_{B} \exp (\xi y) \cos (\xi x-\omega t), & y<0,
\end{array}
$$

where $U_{A}, \Phi_{A}, \Psi_{A}, U_{B}, \Phi_{B}$ and $\Psi_{B}$ are undetermined constants, $\omega$ and $\xi$ are the frequency and wave number, respectively, and

$$
\eta_{A}=\xi \sqrt{1-\frac{v^{2}}{v_{A}^{2}}}>0, \quad \eta_{B}=\xi \sqrt{1-\frac{v^{2}}{v_{B}^{2}}}>0
$$

where $v_{A}=\sqrt{\bar{c}_{A} / \rho_{A}}\left(v_{B}=\sqrt{\bar{c}_{B} / \rho_{B}}\right)$ is the bulk shear wave speed in piezoelectric (piezomagnetic) half-space and $v=\omega / \xi$ is the phase velocity. The stress component $T \equiv T_{z y}(x, y, t)$, electric displacement $D \equiv D_{y}(x, y, t)$ and magnetic induction $B \equiv$ $B_{y}(x, y, t)$ in piezoelectric (piezomagnetic) half-space are denoted by $T_{A}\left(T_{B}\right), D_{A}\left(D_{B}\right)$ and $B_{A}\left(B_{B}\right)$, respectively; and can be expressed by 


$$
\begin{gathered}
T_{A}=\bar{c}_{A} \frac{\partial u_{A}}{\partial y}+e_{A} \frac{\partial \bar{\varphi}}{\partial y}=-\left(\bar{c}_{A} \eta_{A} U_{A} \exp \left(-\eta_{A} y\right)+e_{A} \xi \Phi_{A} \exp (-\xi y)\right) \cos (\xi x-\omega t) \\
D_{A}=-\varepsilon_{A} \frac{\partial \bar{\varphi}_{A}}{\partial y}=\varepsilon_{A} \xi \Phi_{A} \exp (-\xi y) \cos (\xi x-\omega t) \\
B_{A}=-\mu_{A} \frac{\partial \psi_{A}}{\partial y}=\mu_{A} \xi \Psi_{A} \exp (-\xi y) \cos (\xi x-\omega t) \\
T_{B}=\bar{c}_{B} \frac{\partial u_{B}}{\partial y}+f_{B} \frac{\partial \bar{\psi}}{\partial y}=\left(\bar{c}_{B} \eta_{B} U_{B} \exp \left(\eta_{B} y\right)+f_{B} \xi \Psi_{B} \exp (\xi y)\right) \cos (\xi x-\omega t) \\
D_{B}=-\varepsilon_{B} \frac{\partial \varphi_{B}}{\partial y}=-\varepsilon_{B} \xi \Phi_{B} \exp (\xi y) \cos (\xi x-\omega t) \\
B_{B}=-\mu_{B} \frac{\partial \bar{\psi}_{B}}{\partial y}=-\mu_{B} \xi \Psi_{B} \exp (\xi y) \cos (\xi x-\omega t)
\end{gathered}
$$

As mentioned, we consider that the condition for the magneto-electro-mechanical imperfect contact at $y=0$ is given by $[4,11,12]$

$$
\begin{gathered}
T_{A}=T_{B}=K_{u}\left(u_{A}-u_{B}\right), \\
D_{A}=D_{B}=K_{\varphi}\left(\varphi_{B}-\bar{\varphi}_{A}-\frac{e_{A}}{\varepsilon_{A}} u_{A}\right), \\
B_{A}=B_{B}=K_{\psi}\left(\bar{\psi}_{B}+\frac{f_{B}}{\mu_{B}} u_{B}-\varphi_{A}\right) .
\end{gathered}
$$

The first conditions in (7) describe an elastic interface with spring imperfect parameter $K_{u}$, in other words imperfection takes place only in the form of displacement discontinuity. Perfect contact is revealed when the spring imperfect parameter approaches to infinity, while the debonding contact takes place as this imperfect parameter approaches to zero. It may look natural that the mechanical weakening of the interface should also induce the decrease of the electric and/or magnetic contacts due to their magneto-electro-mechanical coupling. The second condition in (7) describes an electric interface with electric capacitor parameter $K_{\varphi}$, i.e. electric potential at the interface is discontinuous. The capacitor represents an interface electric barrier. The capacity of the capacitors determines the degree of the electrical imperfection. When the capacity is equal to zero, the interface is a complete electric barrier. As the capacity increases the degree of electrical imperfection decreases, and perfect electrical interface prevails as the capacity approaches infinity. The third condition in (7) describes a magnetic interface with magnetic inductor parameter $K_{\psi}$, i.e. magnetic potential at the interface is discontinuous. The inductance of the inductors determines the degree of the magnetical imperfection. When the inductance is equal to zero, the interface is a complete magnetic barrier. As the inductance increases the degree of magnetical imperfection decreases, and perfect magnetical interface prevails as the inductance approaches infinity.

A substitution of (2), (3), (5) and (6) into (7) yields to a homogeneous system of linear algebraic equations for $U_{A}, U_{B}, \Phi_{A}, \Phi_{B}, \Psi_{A}$ and $\Psi_{B}$. For non-trivial solutions, the determinant of the principal matrix must be equal to zero, which leads to the following dispersion relation

$$
P_{A} P_{B}+K_{u}\left(P_{A}+P_{B}\right)=0,
$$


where

$$
\begin{aligned}
& P_{A}=\bar{c}_{A}\left(\eta_{A}-\xi \Upsilon_{\varphi} \frac{\bar{K}_{A}^{2}}{\varepsilon_{A}}\right), \quad \bar{K}_{A}^{2}=\frac{e_{A}^{2}}{\bar{c}_{A} \varepsilon_{A}}, \\
& P_{B}=\bar{c}_{B}\left(\eta_{B}-\xi \Upsilon_{\psi} \frac{\bar{K}_{B}^{2}}{\mu_{B}}\right), \quad \bar{K}_{B}^{2}=\frac{f_{B}^{2}}{\bar{c}_{B} \mu_{B}}, \\
& \Upsilon_{\varphi}=\frac{K_{\varphi}}{K_{\varphi}\left(\frac{1}{\varepsilon_{A}}+\frac{1}{\varepsilon_{B}}\right)+\xi}, \quad \Upsilon_{\psi}=\frac{K_{\psi}}{K_{\psi}\left(\frac{1}{\mu_{A}}+\frac{1}{\mu_{B}}\right)+\xi} .
\end{aligned}
$$

Expression (8) determines the phase velocity $v$ and since it changes with frequency, the waves are dispersive. Note that $K_{u} / \bar{c}, K_{\varphi} / \varepsilon$ and $K_{\psi} / \mu$ have dimensions of wave number $(1 / \mathrm{m})$.

Let us consider the following limit cases:

(i) $0<K_{u}<\infty, K_{\varphi} \rightarrow \infty$ and $K_{\psi} \rightarrow \infty$. The interface has perfect electrical and magnetical interactions and partial mechanic interaction. Equation (8) reduces to

$$
\begin{aligned}
& \xi\left(\lambda_{A}-\frac{\varepsilon_{B}}{\varepsilon_{A}+\varepsilon_{B}} \bar{K}_{A}^{2}\right)\left(\lambda_{B}-\frac{\mu_{A}}{\mu_{A}+\mu_{B}} \bar{K}_{B}^{2}\right) \bar{C}_{A} \bar{C}_{B} \\
& \quad+K_{u}\left[\left(\lambda_{A}-\frac{\varepsilon_{B}}{\varepsilon_{A}+\varepsilon_{B}} \bar{K}_{A}^{2}\right) \bar{C}_{A}+\left(\lambda_{B}-\frac{\mu_{A}}{\mu_{A}+\mu_{B}} \bar{K}_{B}^{2}\right) \bar{C}_{B}\right]=0,
\end{aligned}
$$

where $\lambda_{A}=\sqrt{1-v^{2} / v_{A}^{2}}$ and $\lambda_{B}=\sqrt{1-v^{2} / v_{B}^{2}}$. This result appears in [5].

(ii) $K_{u} \rightarrow 0$ and $0<K_{\varphi}, K_{\psi}<\infty$. The interface has partial electric and magnetic interactions but has no mechanical interaction. The two roots of (8) are

$$
v=v_{A} \sqrt{1-\left(\frac{\bar{K}_{A}^{2} \Upsilon_{\varphi}}{\varepsilon_{A}}\right)^{2}}, \quad v=v_{B} \sqrt{1-\left(\frac{\bar{K}_{B}^{2} \Upsilon_{\psi}}{\mu_{B}}\right)^{2}} .
$$

As far as the authors know this is a new result.

(iii) $K_{u} \rightarrow 0$ and $K_{\varphi} \rightarrow \infty$. The interface has perfect electric interaction but has no mechanical interaction (mechanical debonding). Equation (11.1) reduces to

$$
v \equiv v_{A L}=v_{A} \sqrt{1-\frac{\bar{K}_{A}^{4} \varepsilon_{B}^{2}}{\left(\varepsilon_{A}+\varepsilon_{B}\right)^{2}}} .
$$

This result appears in [13].

(iv) $K_{u} \rightarrow 0$ and $K_{\varphi} \rightarrow 0$. In this case, the interface has no mechanical and electrical interaction (electro-mechanical debonding). Equation (11.1) reduces to $v^{2}=v_{A}^{2}$, which do not correspond to an interfacial wave because it is not an evanescent wave towards $\pm \infty$.

(v) $\quad K_{u} \rightarrow 0$ and $K_{\psi} \rightarrow \infty$. The interface has perfect magnetic interaction but has no mechanical interaction. Equation (11.2) reduces to

$$
v \equiv v_{B L}=v_{B} \sqrt{1-\frac{\bar{K}_{B}^{4} \mu_{A}^{2}}{\left(\mu_{A}+\mu_{B}\right)^{2}}} .
$$

This result appears in [13]. 
(vi) $\quad K_{u} \rightarrow 0$ and $K_{\psi}=0$. In this case, the interface has no mechanical and magnetical interaction (magneto-mechanical debonding). Equation (11.2) reduces to $v^{2}=v_{B}^{2}$, which do not correspond to an interfacial wave.

(vii) $K_{u} \rightarrow \infty$ and $0<K_{\varphi}, K_{\psi}<\infty$. The interface has perfect mechanical interaction and partial electric and magnetic interactions. Expression (8) reduces to

$$
\bar{C}_{A} \sqrt{1-\frac{v^{2}}{v_{A}^{2}}}+\bar{C}_{B} \sqrt{1-\frac{v^{2}}{v_{B}^{2}}}=\frac{e_{A}^{2}}{\varepsilon_{A}^{2}} \Upsilon_{\varphi}+\frac{f_{B}^{2}}{\mu_{B}^{2}} \Upsilon_{\psi}
$$

We can see that the left-hand side of (14) is monotonically decreasing with respect to $v$. Therefore, this term reaches its maximum $\bar{C}_{A}+\bar{C}_{B}$ at $v=0$ and its minimum $\bar{C}_{B} \sqrt{1-\left(v_{A} / v_{B}\right)^{2}}$ at $v=v_{A}$ when $v_{A}<v_{B}$. In case that $v_{B}<v_{A}$, we obtain the minimum $\bar{C}_{A} \sqrt{1-\left(v_{B} / v_{A}\right)^{2}}$ at $v=v_{B}$. Consequently, for the existence of the interfacial wave the following conditions must be satisfied:

$$
\begin{gathered}
\bar{C}_{B} \sqrt{1-\frac{v_{A}^{2}}{v_{B}^{2}}}<\frac{e_{A}^{2}}{\varepsilon_{A}^{2}} \Upsilon_{\varphi}+\frac{f_{B}^{2}}{\mu_{B}^{2}} \Upsilon_{\psi}<\bar{C}_{A}+\bar{C}_{B}, \quad v_{A}<v_{B}, \\
\bar{C}_{A} \sqrt{1-\frac{v_{B}^{2}}{v_{A}^{2}}}<\frac{e_{A}^{2}}{\varepsilon_{A}^{2}} \Upsilon_{\varphi}+\frac{f_{B}^{2}}{\mu_{B}^{2}} \Upsilon_{\psi}<\bar{C}_{A}+\bar{C}_{B}, \quad v_{B}<v_{A} .
\end{gathered}
$$

(viii) $\quad K_{u} \rightarrow \infty, K_{\varphi} \rightarrow \infty$ and $K_{\psi} \rightarrow \infty$. The interface has perfect mechanical, electrical and magnetical interaction (perfect bonding). Expression (8) reduces to

$$
\bar{C}_{A} \sqrt{1-\frac{v^{2}}{v_{A}^{2}}}+\bar{C}_{B} \sqrt{1-\frac{v^{2}}{v_{B}^{2}}}=\frac{e_{A}^{2}}{\varepsilon_{A}} \frac{\varepsilon_{B}}{\varepsilon_{A}+\varepsilon_{B}}+\frac{f_{B}^{2}}{\mu_{B}} \frac{\mu_{A}}{\mu_{A}+\mu_{B}} .
$$

This expression appears in $[3,13]$. The conditions for the existence of the interfacial wave are

$$
\begin{gathered}
\bar{C}_{B} \sqrt{1-\frac{v_{A}^{2}}{v_{B}^{2}}}<\frac{e_{A}^{2}}{\varepsilon_{A}} \frac{\varepsilon_{B}}{\varepsilon_{A}+\varepsilon_{B}}+\frac{f_{B}^{2}}{\mu_{B}} \frac{\mu_{A}}{\mu_{A}+\mu_{B}}<\bar{C}_{A}+\bar{C}_{B}, \quad v_{A}<v_{B}, \\
\bar{C}_{A} \sqrt{1-\frac{v_{B}^{2}}{v_{A}^{2}}}<\frac{e_{A}^{2}}{\varepsilon_{A}} \frac{\varepsilon_{B}}{\varepsilon_{A}+\varepsilon_{B}}+\frac{f_{B}^{2}}{\mu_{B}} \frac{\mu_{A}}{\mu_{A}+\mu_{B}}<\bar{C}_{A}+\bar{C}_{B}, \quad v_{B}<v_{A} .
\end{gathered}
$$

(ix) $\quad K_{u} \rightarrow \infty, K_{\varphi} \rightarrow 0$ and $K_{\psi} \rightarrow 0$. The interface has perfect mechanical interaction but has no electrical and magnetical interaction (magnetical and electrical debonding). In this case, $v^{2}=v_{A}^{2}$ and $v^{2}=v_{B}^{2}$.

(x) $K_{u} \rightarrow \infty, K_{\varphi} \rightarrow \infty$ and $K_{\psi} \rightarrow 0$. The interface has perfect mechanical and electrical interaction but has no magnetical interaction. Expression (8) reduces to

$$
\bar{C}_{A} \sqrt{1-\frac{v^{2}}{v_{A}^{2}}}+\bar{C}_{B} \sqrt{1-\frac{v^{2}}{v_{B}^{2}}}=\frac{e_{A}^{2}}{\varepsilon_{A}} \frac{\varepsilon_{B}}{\varepsilon_{A}+\varepsilon_{B}} .
$$


As far as the authors know this is a new result. The conditions for the existence of the interfacial wave are

$$
\begin{gathered}
\bar{C}_{B} \sqrt{1-\frac{v_{A}^{2}}{v_{B}^{2}}}<\frac{e_{A}^{2}}{\varepsilon_{A}} \frac{\varepsilon_{B}}{\varepsilon_{A}+\varepsilon_{B}}<\bar{C}_{A}+\bar{C}_{B}, \quad v_{A}<v_{B}, \\
\bar{C}_{A} \sqrt{1-\frac{v_{B}^{2}}{v_{A}^{2}}}<\frac{e_{A}^{2}}{\varepsilon_{A}} \frac{\varepsilon_{B}}{\varepsilon_{A}+\varepsilon_{B}}<\bar{C}_{A}+\bar{C}_{B}, \quad v_{B}<v_{A} .
\end{gathered}
$$

(xi) $\quad K_{u} \rightarrow \infty, K_{\varphi} \rightarrow 0$ and $K_{\psi} \rightarrow \infty$. The interface has perfect mechanical and magnetical interaction but has no electrical interaction. Expression (8) reduces to

$$
\bar{C}_{A} \sqrt{1-\frac{v^{2}}{v_{A}^{2}}}+\bar{C}_{B} \sqrt{1-\frac{v^{2}}{v_{B}^{2}}}=\frac{f_{B}^{2}}{\mu_{B}} \frac{\mu_{A}}{\mu_{A}+\mu_{B}} .
$$

As far as the authors know this is a new result. The conditions for the existence of the interfacial wave are

$$
\begin{gathered}
\bar{C}_{B} \sqrt{1-\frac{v_{A}^{2}}{v_{B}^{2}}}<\frac{f_{B}^{2}}{\mu_{B}} \frac{\mu_{A}}{\mu_{A}+\mu_{B}}<\bar{C}_{A}+\bar{C}_{B}, \quad v_{A}<v_{B}, \\
\bar{C}_{A} \sqrt{1-\frac{v_{B}^{2}}{v_{A}^{2}}}<\frac{f_{B}^{2}}{\mu_{B}} \frac{\mu_{A}}{\mu_{A}+\mu_{B}}<\bar{C}_{A}+\bar{C}_{B}, \quad v_{B}<v_{A} .
\end{gathered}
$$

In order to illustrate the effect of the mechanical, electrical and magnetical imperfections in the propagation of the wave, we study the interfacial shear horizontal waves between the interface of two half-spaces.

In Figure 1, numerical results of Equations (11.1) and (11.2) are presented to illustrate dispersion curves $v=v(\xi)$. Under the above considerations, the medium A is $\mathrm{BaTiO}_{3}$ with material parameters $c_{A}=4.39 \times 10^{10} \mathrm{~N} / \mathrm{m}^{2}, e_{A}=11.4 \mathrm{C} / \mathrm{m}^{2}, \varepsilon_{A}=9.82 \times 10^{-11} \mathrm{~F} / \mathrm{m}$, $\mu_{A}=5 \times 10^{-6} \mathrm{Ns}^{2} / \mathrm{C}^{2}, \rho_{A}=5.7 \times 10^{3} \mathrm{~kg} / \mathrm{m}^{3}$ and $v_{A}=3165.998 \mathrm{~m} / \mathrm{s}$. The medium $\mathrm{B}$ is $\mathrm{CoFe}_{2} \mathrm{O}_{4}$ and the values of the material constants are $c_{B}=45.3 \times 10^{9} \mathrm{~N} / \mathrm{Am}, f_{B}=$ $550 \mathrm{~N} / \mathrm{m}^{2}, \varepsilon_{B}=0.08 \times 10^{-9} \mathrm{C} / \mathrm{m}^{2}, \mu_{B}=1.57 \times 10^{-4} \mathrm{Ns}^{2} / \mathrm{C}^{2}, \rho_{B}=5.3 \times 10^{3} \mathrm{~kg} / \mathrm{m}^{3}$ and $v_{B}=2985.081 \mathrm{~m} / \mathrm{s}$. The phase velocity given by Equation (11.1) depends on the electric imperfect parameter $\left(K_{\varphi}\right)$. Figure 1a shows the phase velocity for some values of $K_{\varphi} / \varepsilon_{A}(1 / \mathrm{m})=0.2,0.5,1,5,10,20,50,100$. The dispersion curves related to these cases are caught between the phase velocities $v=v_{A}$ for $K_{\varphi} / \varepsilon_{A}(1 / \mathrm{m})=0$ (Case iv) and $v=v_{A L}=3165.992977 \mathrm{~m} / \mathrm{s}$ for $K_{\varphi} / \varepsilon_{A} \rightarrow \infty$ (Case iii). The dispersion curves start from the same initial point because the phase velocity given by the Equation (11.1) for $\xi=0$ is independent of the electric imperfect parameter when it is different from zero. All the curves of Figure 1a are above of $v_{B}$ then the solution of Equation (11.1) does not describe interfacial waves. On the other hand, the phase velocity given by Equation (11.2) depends on the magnetic imperfect parameter $\left(K_{\psi}\right)$. We have taken the following values $K_{\psi} / \mu_{A}(1 / \mathrm{m})=0.2,0.5,1,5,10,20,50,100$ in Figure $1 \mathrm{~b}$ for the illustration. Notice that the curves are restricted between the values $v=v_{B}$ for $K_{\psi} / \mu_{A}(1 / \mathrm{m})=0$ (Case vi) and $v=v_{B L}=2985.078511 \mathrm{~m} / \mathrm{s}$ for $K_{\psi} / \mu_{A} \rightarrow \infty$ (Case v). Therefore, the solution given by Equation (11.2) represents interfacial waves. 
(a)
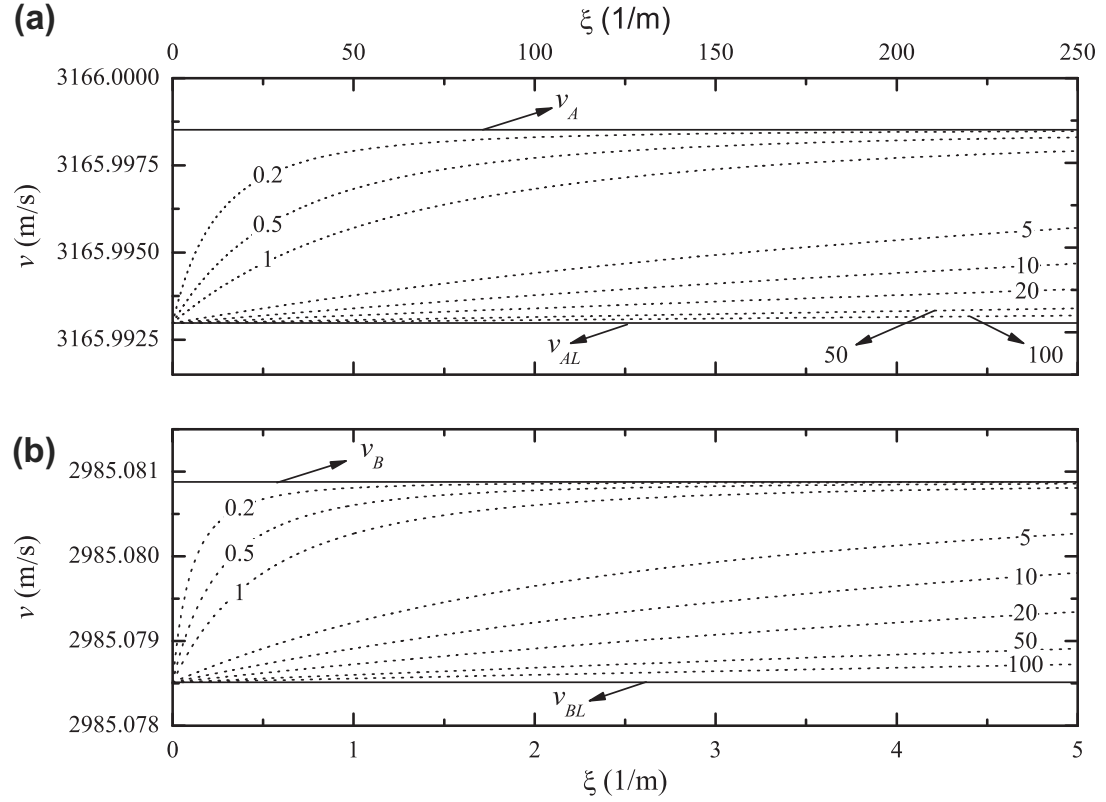

Figure 1. Variation of the phase velocity $(v)$ vs. wave number $(\xi)$ for the elastic imperfect parameter equal to zero and different values $K_{\varphi} / \varepsilon_{A}(1 / \mathrm{m})=0.2,0.5,1,5,10,20,50,100$ (a) and $K_{\psi} / \mu_{A}(1 / \mathrm{m})=0.2,0.5,1,5,10,20,50,100(\mathrm{~b})$.

Now, our intention is to study numerically the solution of Equation (14) for perfect mechanic with electric and magnetic imperfect contacts (Case vii). The condition given by Equation (16) fails for the above used materials and therefore there are not interfacial waves (see Table 1). Since the presence of interfacial shear horizontal waves between the interface of piezoelectric and piezomagnetic half-spaces for perfect mechanic with electric and magnetic imperfect contacts is not manifested with the conventional materials ( $\mathrm{BaTiO}_{3}$ and $\mathrm{CoFe}_{2} \mathrm{O}_{4}$ ), we analyse numerically the interfacial shear horizontal waves between two half-spaces with speeds very near (where the left-hand side of condition (16) is very small). For that purpose, we consider the medium $\mathrm{A}$ as $\mathrm{BaTiO}_{3}$ and we choose a medium $\mathrm{B}$ (fictitious medium) under the following condition: the speeds are $v_{B}=\gamma v_{A}$, which can be obtained from the relations $c_{B}=\gamma^{2} c_{A}, f_{B}^{2} / \mu_{B}=\gamma^{2} e_{A}^{2} / \varepsilon_{A}$ and $\rho_{B}=\rho_{A}$, where $\gamma$ is a number close to 1 . Under the aforementioned conditions, the material parameters of the medium $\mathrm{B}$ are $\varepsilon_{B}=\varepsilon_{A}, \mu_{B}=\mu_{A}, \rho_{B}=\rho_{A}, c_{B}=\gamma^{2} c_{A}$ and $f_{B}=\gamma e_{A} \sqrt{\mu_{B} / \varepsilon_{A}}$. In the calculations, we use $\gamma=0.999$. The dispersion curves $v=v(\xi)$ obtained from Equation (14) are shown in Figure 2.

Firstly, the value $K_{\psi} / \mu_{A}(1 / \mathrm{m})=10^{4}$ is fixed (magnetic perfect contact) and the electric parameter is varied for different values $K_{\varphi} / \varepsilon_{A}(1 / \mathrm{m})=0.5,1,5,10,20,30,50,10^{2}$, $10^{3}, 10^{4}$. Secondly, the electric imperfection is selected as $K_{\varphi} / \varepsilon_{A}(1 / \mathrm{m})=10^{4}$ (electric perfect contact) and the magnetic imperfection takes different values, for instance, $K_{\psi} / \mu_{A}(1 / \mathrm{m})=0.5,1,5,10,20,30,50,10^{2}, 10^{3}, 10^{4}$. The dispersion curves are very close each other for the particular case $K_{\varphi} / \varepsilon_{A}=K_{\psi} / \mu_{A}$ and they are monotonically 
Table 1. Numerical proof of the condition given in Equation (16) for $\mathrm{BaTiO}_{3} / \mathrm{CoFe}_{2} \mathrm{O}_{4}$ half-spaces.

\begin{tabular}{lccccc}
\hline$\xi(1 / \mathrm{m})$ & $\frac{K_{\varphi}}{\varepsilon_{A}}(1 / \mathrm{m})$ & $\frac{K_{\psi}}{\mu_{A}}(1 / \mathrm{m})$ & $\bar{C}_{A} \sqrt{1-\frac{v_{B}^{2}}{v_{A}^{2}}}\left(\mathrm{~N} / \mathrm{m}^{2}\right)$ & $\frac{e_{A}^{2}}{\varepsilon_{A}^{2}} \Upsilon_{\varphi}+\frac{f_{B}^{2}}{\mu_{B}^{2}} \Upsilon_{\psi}\left(\mathrm{N} / \mathrm{m}^{2}\right)$ & $\bar{C}_{A}+\bar{C}_{B}\left(\mathrm{~N} / \mathrm{m}^{2}\right)$ \\
\hline 10 & 0.5 & $10^{4}$ & $1.9037 \times 10^{10}$ & $1.5152 \times 10^{8}$ & $1.0436 \times 10^{11}$ \\
10 & 1.0 & $10^{4}$ & $1.9037 \times 10^{10}$ & $1.5841 \times 10^{8}$ & $1.0436 \times 10^{11}$ \\
10 & 5 & $10^{4}$ & $1.9037 \times 10^{10}$ & $1.6470 \times 10^{8}$ & $1.0436 \times 10^{11}$ \\
10 & 10 & $10^{4}$ & $1.9037 \times 10^{10}$ & $1.6555 \times 10^{8}$ & $1.0436 \times 10^{11}$ \\
10 & 20 & $10^{4}$ & $1.9037 \times 10^{10}$ & $1.6597 \times 10^{8}$ & $1.0436 \times 10^{11}$ \\
10 & 30 & $10^{4}$ & $1.9037 \times 10^{10}$ & $1.6612 \times 10^{8}$ & $1.0436 \times 10^{11}$ \\
10 & 50 & $10^{4}$ & $1.9037 \times 10^{10}$ & $1.6623 \times 10^{8}$ & $1.0436 \times 10^{11}$ \\
10 & $10^{2}$ & $10^{4}$ & $1.9037 \times 10^{10}$ & $1.6632 \times 10^{8}$ & $1.0436 \times 10^{11}$ \\
10 & $10^{3}$ & $10^{4}$ & $1.9037 \times 10^{10}$ & $1.6640 \times 10^{8}$ & $1.0436 \times 10^{11}$ \\
10 & $10^{4}$ & $10^{4}$ & $1.9037 \times 10^{10}$ & $1.6641 \times 10^{8}$ & $1.0436 \times 10^{11}$ \\
\hline
\end{tabular}

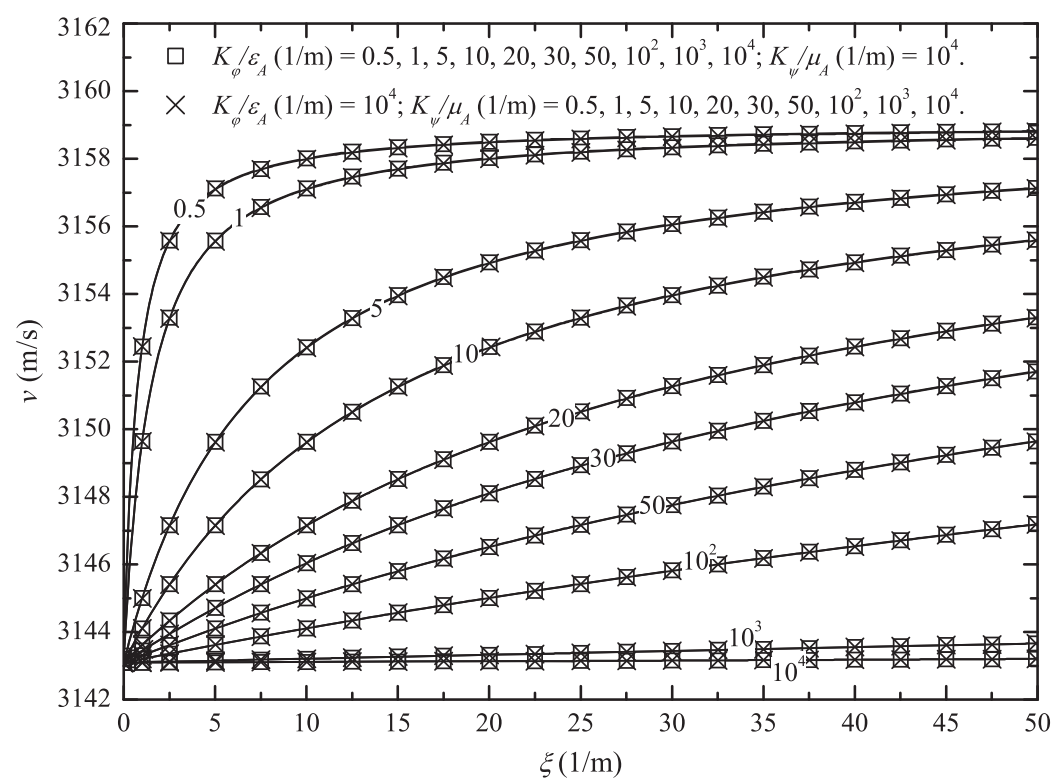

Figure 2. Variation of the phase velocity $(v)$ vs. wave number $(\xi)$ for the elastic imperfect parameter equal to $K_{u} / c_{A} \rightarrow \infty$ and different values of electric and magnetic imperfect parameters.

increasing functions. These curves describe interfacial waves since they are inferior to the velocity of the medium $B$.

Finally, a strongly influence of the mechanic, electric and magnetic imperfection adherences is observed on the presence of interfacial waves between piezoelectric and piezomagnetic half-spaces. 


\section{Acknowledgements}

The authors wish to acknowledge the CoNaCyT/México projects No. 129658, 82474 and the DGAPA UNAM/México project PAPIIT IN 119509-3. This work was partially written while R. RodríguezRamos was visiting the Laboratoire de Mécanique et d'Acoustique (LMA-CNRS), Marseille, France. Thanks to Ecole Central Marseille for the support given for the visit.

\section{References}

[1] Z. Yang and J. Yang, Int. J. Appl. Electromag. Mech. 29 (2009) p.101.

[2] L. Nan, J. Yang, Z.-H. Qian and S. Hirose, Int. J. Eng. Sci. 48 (2010) p.151.

[3] A.K. Soh and J.X. Liu, Phil. Mag. Lett. 86 (2006) p.31.

[4] H. Fan, J. Yang and L. Xu, Appl. Phys. Lett. 88 (2006) p.203509.

[5] A. Melkumyan and Y.-W. Mai, Phil. Mag. 88 (2008) p.2965.

[6] Y. Huang, X.-F. Li and K.Y. Lee, Phil. Mag. Lett. 89 (2009) p.95.

[7] A.N. Darinskii and M. Weihnacht, Wave Motion 43 (2005) p.67.

[8] Y.-Z. Bai, Y.-S. Wang and G.-L. Yu, Int. J. Eng. Sci. 4 (2007) p.1017.

[9] J.A. Otero, R. Rodriguez-Ramos, J. Bravo-Castillero, A.R. Aguiar and G. Monsivais, J. Mech. Mat. Struct. 6 (2011) p.969.

[10] J.A. Otero, R. Rodriguez-Ramos, J. Bravo-Castillero and G. Monsivais, Phil. Mag. Lett. 92 (2012) p.534.

[11] H.M. Shodja, S.M. Tabatabaei and M.T. Kamali, Int. J. Solids Struct. 44 (2007) p.6361.

[12] W.-H. Sun, G.-L. Ju, J.-W. Pan and Y.-D. Li, Ultrasonic 51 (2011) p.831.

[13] Y. Huang and X.F. Li, Ultrasonics 50 (2010) p.750. 